\title{
Large Penetrating Keratoplasty in the Management of Keratoglobus: A Case Report
}

\author{
Lamprini Papaioannou, Miltiadis Papathanassiou \\ Cornea Clinic, 2nd Ophthalmology Department, Attikon University Hospital, Athens, Greece \\ Email: papathanassiou1@gmail.com
}

Received 19 November 2015; accepted 23 February 2016; published 26 February 2016

Copyright (C) 2016 by authors and Scientific Research Publishing Inc.

This work is licensed under the Creative Commons Attribution International License (CC BY). http://creativecommons.org/licenses/by/4.0/

(c) (i) Open Access

\begin{abstract}
Background: Keratoglobus is a rare noninflammatory corneal disorder characterized by diffuse corneal thinning and globular protrusion of the cornea. Surgical management of keratoglobus is challenging and the standard method has not yet been defined. Aim: To present the role of large penetrating keratoplasty (PK) in the management of keratoglobus. Case Presentation: A 29-year-old male patient with bilateral keratoglobus presented with acute corneal hydrops in his right eye following extensive Descemet's membrane rupture, with a visual acuity in this eye limited to hand movement. Peripheral cornea was extremely thin and blue sclera was present. Acute hydrops was managed conservatively at this stage and two months later large PK was performed in the right eye using $9.5 \mathbf{~ m m}$ diameter graft over a $9 \mathrm{~mm}$ patient's cornea trephination. Minor aqueous leakage was seen on the first postoperative day, managed with 2 more interrupted 10.0 nylon sutures. No further complications were noticed and postoperative course was uneventful. Fifteen months postoperatively the graft was clear and best corrected visual acuity was $20 / 60$. Conclusions: Large penetrating keratoplasty has an important role in the management of keratoglobus, in cases where peripheral tuck-in lamellar keratoplasty or epikeratoplasty present serious intraoperative difficulties in host lamellar dissection and in stabilizing the graft due to extensive peripheral corneal and scleral thinning.
\end{abstract}

\section{Keywords}

Keratoglobus, Large Penetrating Keratoplasty, Corneal Ectasia

\section{Introduction}

Keratoglobus is a rare noninflammatory corneal disorder characterized by diffuse corneal thinning from limbus

How to cite this paper: Papaioannou, L. and Papathanassiou, M. (2016) Large Penetrating Keratoplasty in the Management of Keratoglobus: A Case Report. Open Journal of Ophthalmology, 6, 51-55. http://dx.doi.org/10.4236/ojoph.2016.61007 
to limbus, commonly maximal at the periphery and globular protrusion of the cornea [1]. Keratoglobus is primarily considered a congenital disorder, although acquired forms of keratoglobus have been reported. The congenital form of the disorder is always bilateral and it is assumed to be autosomal recessive but to our knowledge the inheritance pattern has not yet been defined [2]. It has also been associated with disorders of the connective tissue such as Ehlers-Danlos syndrome, Marfan syndrome, and Rubinstein-Taybi syndrome, with the former being the most common association. Other systemic associations include Leber's congenital amaurosis, Syphilis and Thyroid eye disease [1]. Aquired keratoglobus has been described in idiopathic orbital inflammation, possibly caused by anterior segment ischaemia and in vernal keratoconjuctivitis and chronic marginal blepharitis, possibly attributable to frequent eye rubbing. The most common ocular feature associated with keratoglobus is blue sclera caused by a thinned and more transparent sclera, maximally at the ciliary body. Patients with keratoglobus have clear corneas with normal diameter and the main cause of poor vision is high myopia with irregular astigmatism caused by excessive corneal thinning and protrusion. Corneal thinning and fragility can result in corneal perforations, either spontaneous or following minor trauma. Spontaneous tears in Descemet's membrane may also occur, resulting in acute hydrops [1] [3].

Surgical management of keratoglobus is challenging and the standard method has not yet been defined, considering the rarity and the special features of the condition, mainly including the corneal thinning from limbus to limbus. Several modifications of conventional penetrating and lamellar keratoplasty procedures have been reported, aiming to overcome the special difficulties and risk factors. We present the role of large penetrating keratoplasty (PK) in the management of the condition.

\section{Case Presentation}

A 29-year old male patient with known history of bilateral keratoglobus presented to the Cornea Clinic with pain, photophobia and blurred vision in his right eye of two days duration. Best corrected visual acuity was limited to hand movement in the right eye and 20/120 in left eye. The diagnosis of bilateral keratoglobus was established with corneal topography (Oculus Pentacam). Slit lamp examination revealed acute corneal hydrops caused by extensive Descemet's membrane rupture in his right eye (Figure 1), excessive thinning in the juxtalimbal periphery and coexisting blue sclera in both eyes (Figure 2). Intraocular pressure was 11 and $12 \mathrm{mmHg}$ in the right and left eye respectively and further eye examination was unremarkable. No further systemic associations were present.

Acute hydrops was managed conservatively with topical hypertonic agents at this stage as the extent of the break made the use of intracameral gas for reattachment extremely difficult. Two months later, a large PK was performed in the right eye for visual rehabilitation. A $9.5 \mathrm{~mm}$ diameter graft was used over a $9 \mathrm{~mm}$ patient's cornea trephination. 22 interrupted 10.0 nylon sutures were placed and the procedure was uneventful.

Minor aqueous leak between 2nd and 3rd hour was noticed on the first postoperative day, managed with two additional interrupted 10.0 nylon sutures. No further complications were noticed. Fifteen months postoperatively the graft remained clear and best corrected visual acuity was 20/60 (Figure 3).

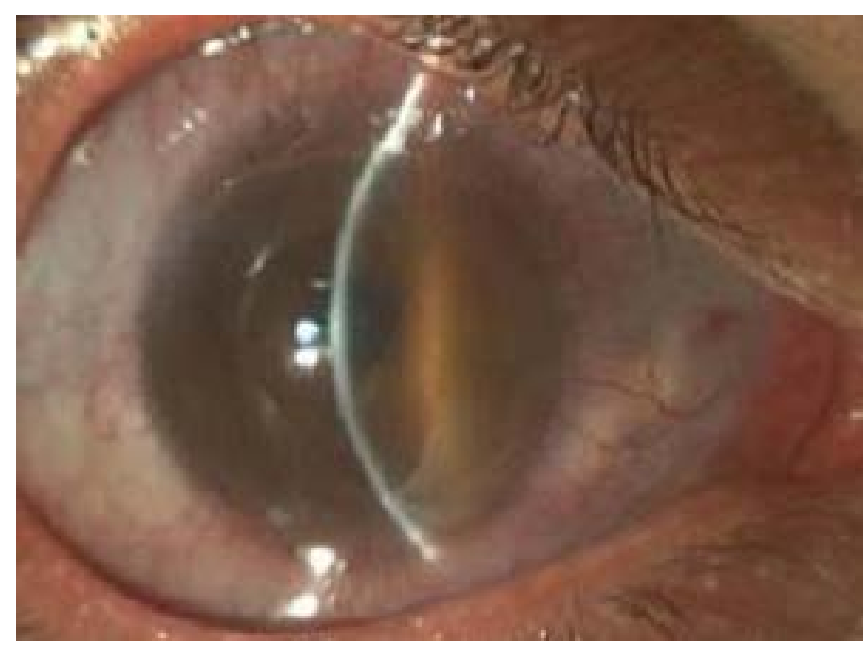

Figure 1. Extensive descemet’s membrane rupture. 


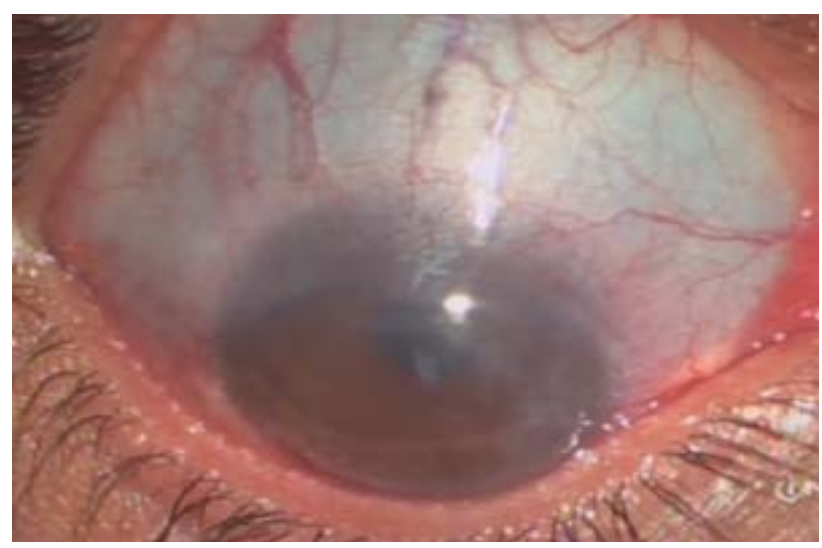

Figure 2. Co-existing blue sclera was present in our patient.

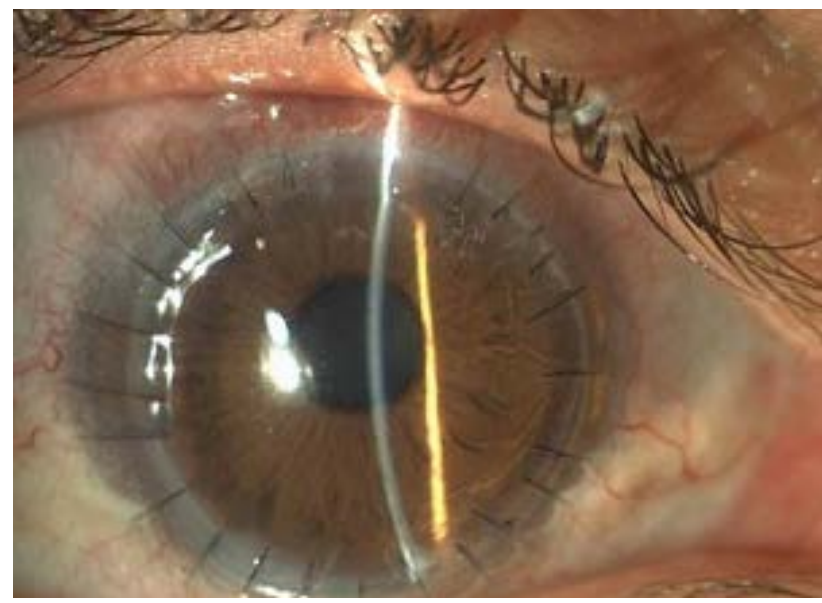

Figure 3. The $9.5 \mathrm{~mm}$ diameter graft remained clear 15 months postoperatively.

\section{Discussion}

The features of keratoglobus as discussed above, make the management of the condition challenging. Regarding the management of acute hydrops, it can be both conservative and invasive. Conventional treatment includes bandage contact lens, topical hypertonic saline and cycloplegics [3]. More invasive is the use of intracameral gas to tamponade the detached descemet's membrane to the posterior surface of the corneal stroma [4] [5]. Regarding the surgical management of keratoglobus, no golden standard has been defined. Conventional penetrating keratoplasty presents difficulties in wound closure, due to excessive mid-peripheral corneal thinning and graft to host thickness disparity. Therefore, an alternative option of penetrating keratoplasty has been described, using large limbus to limbus donor corneal grafts to avoid placement of the graft-host junction at the thinned mid-periphery [6]. Thereby, wound healing and tectonic stability are promoted, but the immunological privilege of corneal transplant is lost, while limbal stem cells are disrupted [7]. Several modifications of lamellar keratoplasty have also been described in the literature. Epikeratoplasty is such a technique that uses a donor graft, denuded from descemet's membrane, placed over the host cornea after posterior dissection of the conjunctiva [8]. The graft is sutured to the sclera and thus epikeratoplasty has the advantantage of avoiding suturing to the thin corneal periphery, but this advantage is limited in cases with coexisting scleral thinning. On the other hand, this technique disrupts limbal stem cells and could result in persistant epithelial defects. Promising were the results of a modified epikeratoplasty technique, described by Javadi et al., aiming to avoid limbal stem cell damage, by creating a 360 degree peripheral lamellar intrastromal pocket in the host cornea for the insertion of the donor corneo-scleral button [9]. Epikeratoplasty followed by secondary penetrating keratoplasty for visual rehabilitation has also been reported [10]. An alternative technique is "Tuck-in" lamellar keratoplasty (TILK) that in- 
volves a central lamellar dissection along with a peripheral intrastromal pocket, where the peripheral donor flange is tucked in [11]. Further novel techniques have been reported, such as the use of an allograft corneoscleral ring around the limbus to support the mid-peripheral thinned cornea, described by Kanellopoulos and Pentacam-based big bubble deep anteriorlamellar keratoplasty, described by Riss et al. [12] [13] More recently, Karimian et al. described a limbal stem cell-sparing lamellar keratoplasty (LSCS-LKP) and Lockington et al. described a lamellar keratoplasty with a pleat creation to address the abnormal white-to-white diameter [14] [15].

In our case, excessive corneal thinning and scleral thinning were considered as high risk factors for serious intraoperative difficulties in host lamellar dissection and in stabilizing the graft in a tuck-in lamellar keratoplasty or epikeratoplasty. Additionally, the presence of an extensive descemet's membrane rupture would require a secondary PK for visual rehabilitation. Therefore large PK was felt a safer approach, as it allowed the replacement of the ruptured descemet's membrane, while the immune disadvantage of a limbus to limbus PK was limited due to the smaller size of the graft $(9.5 \mathrm{~mm})$. Furthermore, limbal stem cell and angle structure disruption was avoided. On the other hand, the graft suturing was still challenging and more sutures were placed compared to the standard PK technique. However, a minor aqueous leak was noticed on the first postoperative day, managed with two additional sutures. No further complications were noticed. To our knowledge, large PK has already been reported in a keratoglobus case by Kodjikian et al., using a mid-sized graft of $9 \mathrm{~mm}$ diameter placed eccentrically [16]. However, since no standard method has yet been defined and no large series regarding the surgical management of keratoglobus are available in the literature, one additional report could contribute to this objective.

\section{Conclusion}

In conclusion, the rarity of the condition limits the ability of comparative studies or large case series. Therefore, reports of surgeons' experience, obtained even by single cases could be helpful in drawing conclusions for the options of keratoglobus surgical management. In this perspective, we believe that large penetrating keratoplasty has an important role in keratoglobus management, especially in cases with descemet's membrane rupture and co-existing blue sclera.

\section{References}

[1] Wallang, B.S. and Das, S. (2013) Keratoglobus. Eye, 27, 1004-1012. http://dx.doi.org/10.1038/eye.2013.130

[2] Pouliquen, Y., Dhermy, P., Espinasse, M.A. and Savoldelli, M. (1985) Keratoglobus. Journal Français d'Ophtalmologie, 8, 43-45.

[3] Grewal, S., Laibson, P.R., Cohen, E.J. and Rapuano, C.J. (1999) Acute Hydrops in the Corneal Ectasias: Associated Factors and Outcomes. Transactions of the American Ophthalmological Society, 97, 187-198.

[4] Panda, A., Aggarwal, A., Madhavi, P., Wagh, V.B., Dada, T., Kumar, A., et al. (2007) Management of Acute Corneal Hydrops Secondary to Keratoconus with Intracameral Injection of Sulfur Hexafluoride (SF6). Cornea, 26, 1067-1069. http://dx.doi.org/10.1097/ICO.0b013e31805444ba

[5] Shah, S.G., Sridhar, M.S. and Sangwan, V.S. (2005) Acute Corneal Hydrops Treated by Intracameral Injection of Perfluoropropane (C3F8) Gas. American Journal of Ophthalmology, 139, 368-370. http://dx.doi.org/10.1016/j.ajo.2004.07.059

[6] Cowden, J.W., Copeland, R.A. and Schneider, M.S. (1989) Large Diameter Therapeutic Penetrating Keratoplasties. Refractive \& Corneal Surgery, 5, 244-248.

[7] Khodadoust, A.A. and Silverstein, A.M. (1972) Studies on the Nature of the Privilege Enjoyed by Corneal Allografts. Investigative Ophthalmology, 11, 137-148.

[8] Cameron, J.A., Cotler, J.B., Risco, J.M. and Alvarez, H. (1991) Epikeratoplasty for Keratoglobus Associated with Blue Sclera. Ophthalmology, 98, 446-452. http://dx.doi.org/10.1016/S0161-6420(91)32271-1

[9] Javadi, M.A., Kanavi, M.R., Ahmadi, M. and Yazdani, S. (2007) Outcomes of Epikeratoplasty for Advanced Keratoglobus. Cornea, 26, 154-157. http://dx.doi.org/10.1097/01.ico.0000244878.38621.fc

[10] Jones, D.H. and Kirkness, C.M. (2001) A New Surgical Technique for Keratoglobus-Tectonic Lamellar Keratoplasty Followed by Secondary Penetrating Keratoplasty. Cornea, 20, 885-887. http://dx.doi.org/10.1097/00003226-200111000-00022

[11] Kaushal, S., Jhanji, V., Sharma, N., Tandon, R., Titiyal, J.S. and Vajpayee, R.B. (2008) “Tuck-In” Lamellar Keratop- 
lasty (TILK) for Corneal Ectasias Involving Corneal Periphery. British Journal of Ophthalmology, 92, 286-290. http://dx.doi.org/10.1136/bjo.2007.124628

[12] Kanellopoulos, A.J. and Pe, L.H. (2005) An Alternative Surgical Procedure for the Management of Keratoglobus. Cornea, 24, 1024-1026. http://dx.doi.org/10.1097/01.ico.0000157411.31566.a7

[13] Riss, S., Heindl, L.M., Bachmann, B.O., Kruse, F.E. and Curseifen, C. (2012) Pentacam-Based Big Bubble Deep Anterior Lamellar Keratoplasty in Patients with Keratoconus. Cornea, 31, 627-632. http://dx.doi.org/10.1097/ICO.0b013e31823f8c85

[14] Karimian, F., Baradaran-Rafii, A., Faramarzi, A. and Akbari, M. (2014) Limbal Stem Cell-Sparing Lamellar Keratoplasty for the Management of Advanced Keratoglobus. Cornea, 33, 105-108. http://dx.doi.org/10.1097/ICO.0b013e3182a9b1ac

[15] Lockington, D. and Ramaesh, K. (2015) Use of a Novel Lamellar Keratoplasty with Pleat Technique to Address the Abnormal White-to-White Diameter in Keratoglobus. Cornea, 34, 239-242. http://dx.doi.org/10.1097/ICO.0000000000000315

[16] Kodjikian, L., Baillif, S., Burillon, C., Grange, J.D. and Garweg, J.G. (2004) Keratoglobus Surgery: Penetrating Keratoplasty Redux. Acta Ophthalmologica Scandinavica, 82, 625-627. http://dx.doi.org/10.1111/j.1600-0420.2004.00271.x 